\title{
Study of the Effect of Air to Fuel ratio Parameter on the Organophosphorus - Pesticide Analysis by GC-FPD
}

\author{
A. Y. Kilany, M. A. Elsayed, M. K. Abd Elmegid, M. S. Fayed \\ Egyptian Armed Forces, Cairo, Egypt \\ Tel. +201141750544; Fax: +222621918
}

E-mail address: aboelfotoh@gmail.com

\begin{abstract}
In the present contribution, sensitive and precise method for the quantification of Organophosphorus / Pesticides (Malathion and Dimethoate) in nanograms range has been developed. The performance of flame photometric detector (FPD), a selective detector (P\&S-mode) that can be used in the analysis of organophosphorus compound, is evaluated in terms of sensitivity, selectivity and reproducibility. The performance of flame photometric detector was strongly depending on the absolute and relative flow rate of air and hydrogen gases. The optimum air-to-fuel ratio for detection of Malathion and Dimethoate was 0.4 and 0.3 (FPD-P mode). At this ratio, low picogram amounts of phosphor can be detected accurately $(0.18 \mathrm{pgP})$ with a wide linear dynamic range of $0.18 \mathrm{pgP}$ to 298 ngP. While, the optimum air-to-fuel ratio, for detection of Malathion and Dimethoate was 0.6 (FPD-S mode). In addition to, the method is precise with $4.5 \%$ relative standard deviation (RSD). In conclusion, it could be proposed that this procedure can be recommended as a suitable method for the quantification of Malathion and Dimethoate in cases of acute poisoning.
\end{abstract}

Keywords: Sensitivity; Minimum Detectability; Dynamic Range; Linear Range; Organophosphorus pesticides; Malathion; Dimethoate; GC/FPD

\section{INTRODUCTION}

Organophosphorus (OP) pesticides are widely used in agriculture as insecticides due to their high activity, low bioaccumulation and moderately rapid degradation in the environment. Nevertheless, a certain amount of the pesticides used is transferred in the surface runoff and subsurface drainage from agricultural land. This can cause a spectrum of toxic effects on aquatic organisms and human beings. At the mammalian level, organophosphorus pesticides represent a serious risk because of their irreversible binding to the active site of acetyl cholinesterase (AChE). This leads to hindered hydrolysis of the acetylcholine (AChE) resulting in the dysfunction of the transmission of nerve impulses with poisoning effects and nervous diseases. The measurements of OP compounds are classically done using chromatographic techniques [1-6].

Malathion S-[1,2-di(ethoxycarbonyl)ethyl] dimethyl phosphorothiolothionate is a member of a general class of organophosphorus or organophosphate (OP) pesticides. Malathion is not only used to control insects on fruits and vegetables, but is also employed in 
the management of mosquitoes. This non-systemic pesticide kills insects by direct contact or through vapor action which binds irreversibly to cholinesterase [7]. The oral LD50 values for Malathion in laboratory rodents were $1000-10000 \mathrm{mg} / \mathrm{kg}$ of body weight, the observed differences probably being due to impurities [8]. The chemical structure of Malathion is shown in Figure 1 [9].

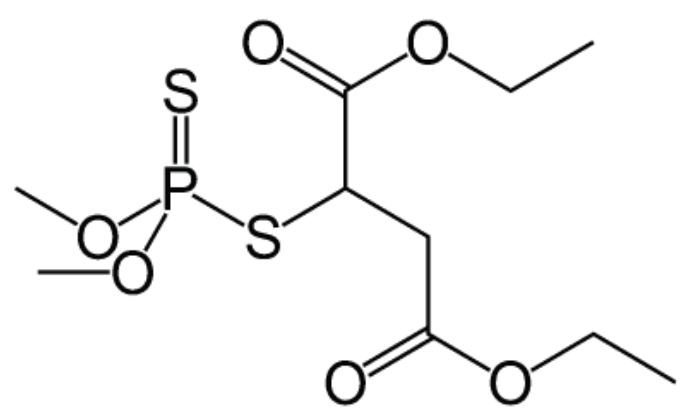

Fig. 1. The Chemical Structure of Malathion.

Dimethoate is (O,O-dimethyl S-methyl-carbamoyl-methyl phosphorodithioate) or it is O,O-dimethylS-[2-(methylamino)-2-oxoethyl] Dithiophosphate. The chemical structure of Dimethoate is shown in Figure 2 [9]. Dimethoate major uses are an organophosphorus insecticide with a contact and systemic poison action. It was introduced in 1956 and is produced in many countries. It is a general use chemical for use against a broad range of insects in agriculture and also for the control of the housefly [10].

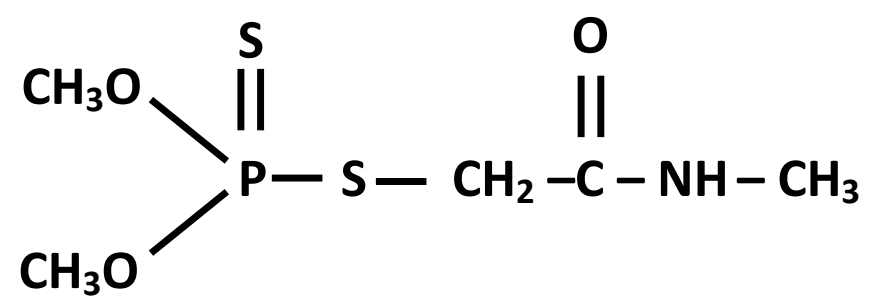

Fig. 2. The Chemical Structure of Dimethoate.

The Flame Photometric Detector (FPD) detects compounds by burning those compounds in a flame and sensing the increase of light emission from the flame during that combustion process. Therefore, the FPD is a flame optical emission detector comprised of; a hydrogen-air flame, an optical window for viewing emissions generated in the flame, an optical filter for spectrally selecting the wavelengths of light detected, a photomultiplier tube for measuring the intensity of light emitted and an electrometer for measuring the current output of the photomultiplier. The sensitivity and specificity of the FPD are strongly dependent on the absolute and relative flow rates of hydrogen and air. The optimum hydrogen and air flow rates depend on the detailed configuration of the flame burner. For some FPD designs, the flow which is optimum for phosphorus detection is not the same as the flows which is optimum for sulfur detection. Also, the flow which is optimum for one sample compound may not necessarily be optimum for another sample compound [11]. 


\section{EXPERIMENTAL}

\section{1. Reagents and solutions}

Malathion and Dimethoate were purchased from Nasr Company for intermediate chemicals, Egypt with purity $>95 \%$. Stock standard solutions were prepared by accurately weighing $0.3 \mathrm{~g}$ of pure material. Malathion and Dimethoate were dissolved in pesticidequality benzene and diluted to a volume of $25 \mathrm{~mL}$ in volumetric flask. Stock standard solutions were transferred into TFE-fluorocarbon-sealed screw-cap vials, stored at $4{ }^{\circ} \mathrm{C}$ and protected from light. A set of test samples were prepared by dilution from stock standard solutions, covering a wide range of concentrations. A fixed volume $(1 \mu \mathrm{L})$ of each concentration of the test substance was injected, the height $(\mathrm{H})$ and integrated peak area (A) of the resultant Gaussian or chromatographic peaks were measured. Frequently stock standard solutions were checked for signs of degradation or evaporation.

\section{2. Instrumentation and analysis conditions}

Agilent, 7890A gas chromatograph, Autosampler (7693-series) with a split/ Splitless injector system and a flame photometric detection with phosphorus filter were used. Ultra pure nitrogen $(99.9999 \%)$ at 25 psi constant pressure was passed through a molecular sieve trap and an oxygen trap was used as the carrier gas. The injection port was held at $200{ }^{\circ} \mathrm{C}$ and used in the splitless mode. Separation was carried out on a HB-5, $30 \mathrm{~m}$ x $0.32 \mathrm{~mm}$ capillary column with a $0.25 \mu \mathrm{m}$ stationary film thickness (Agilent Technologies). Balance: Analytical, capable of accurately weighing to the nearest $0.0001 \mathrm{~g}$. The column temperature was maintained at $60{ }^{\circ} \mathrm{C}$ for $1 \mathrm{~min}$ and then programmed at $20^{\circ} \mathrm{C} / \mathrm{min}$ to $250{ }^{\circ} \mathrm{C}$, and held for 5 min. The total analysis time was $15.5 \mathrm{~min}$. The volume of sample injected in splitless mode was $1.00 \mu \mathrm{L}$. Absolute and relative flow rate of air and hydrogen used for flame photometric detector were studied from 0.2-1.2 air-to-hydrogen ratio.

\section{RESULTS AND DISCUSSIONS}

\section{1. Influence of air-to-hydrogen flow rates ratio on FPD performance:}

The intensity and wavelength of light emitted from the FPD flame depends on the geometric configuration of the flame burner and on the absolute and relative flow rates of gases supplied to the detector. By judicious selection of burner geometry and gas flow rates, the FPD flame is usually designed to selectively enhance optical emissions from certain types of molecules while suppressing emissions from other molecules [11]. Figures 3 and 4 show the effect of relative air-to-hydrogen ratio flow rates on FPD performance at constant organphosphorus concentration $(1 \mu \mathrm{L}$ injection). The result show that the optimum condition for detector response with malathion is 0.4 while for dimethoate is 0.3 . The make-up gas flow rate was found to have only a marginal effect on the FPD performance. In all further experiments a make-up flow of $60 \mathrm{ml} / \mathrm{min}$ was used.

The detector temperature was set at $250{ }^{\circ} \mathrm{C}$. The sensitivity of the detector was found to be maximum at 0.4 air-to-fuel ratio for malathion and 0.3 with dimethoate. The chromatograms of detector responses against air-to- $\mathrm{H}_{2}$ ratio for malathion and dimethoate are shown in Figure 5 and 6 as 3D figure, respectively. 


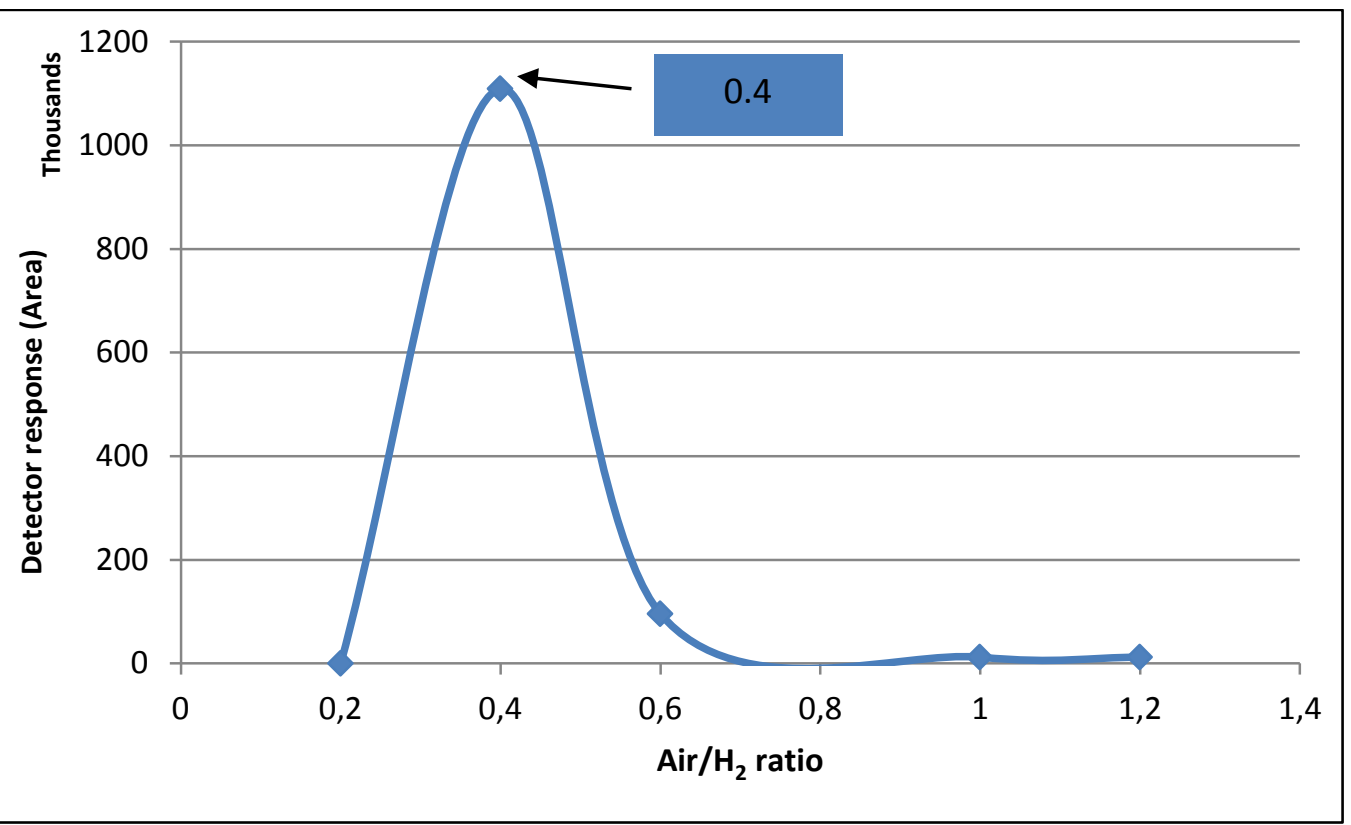

Fig. 3. Detector responses at different air-to-hydrogen ratio GC-FPD-P mode for Malathion, measured at GC operating condition.



Fig. 4. Detector responses at different air-to-hydrogen ratio GC-FPD-P mode for Dimethoate, measured at GC operating condition. 


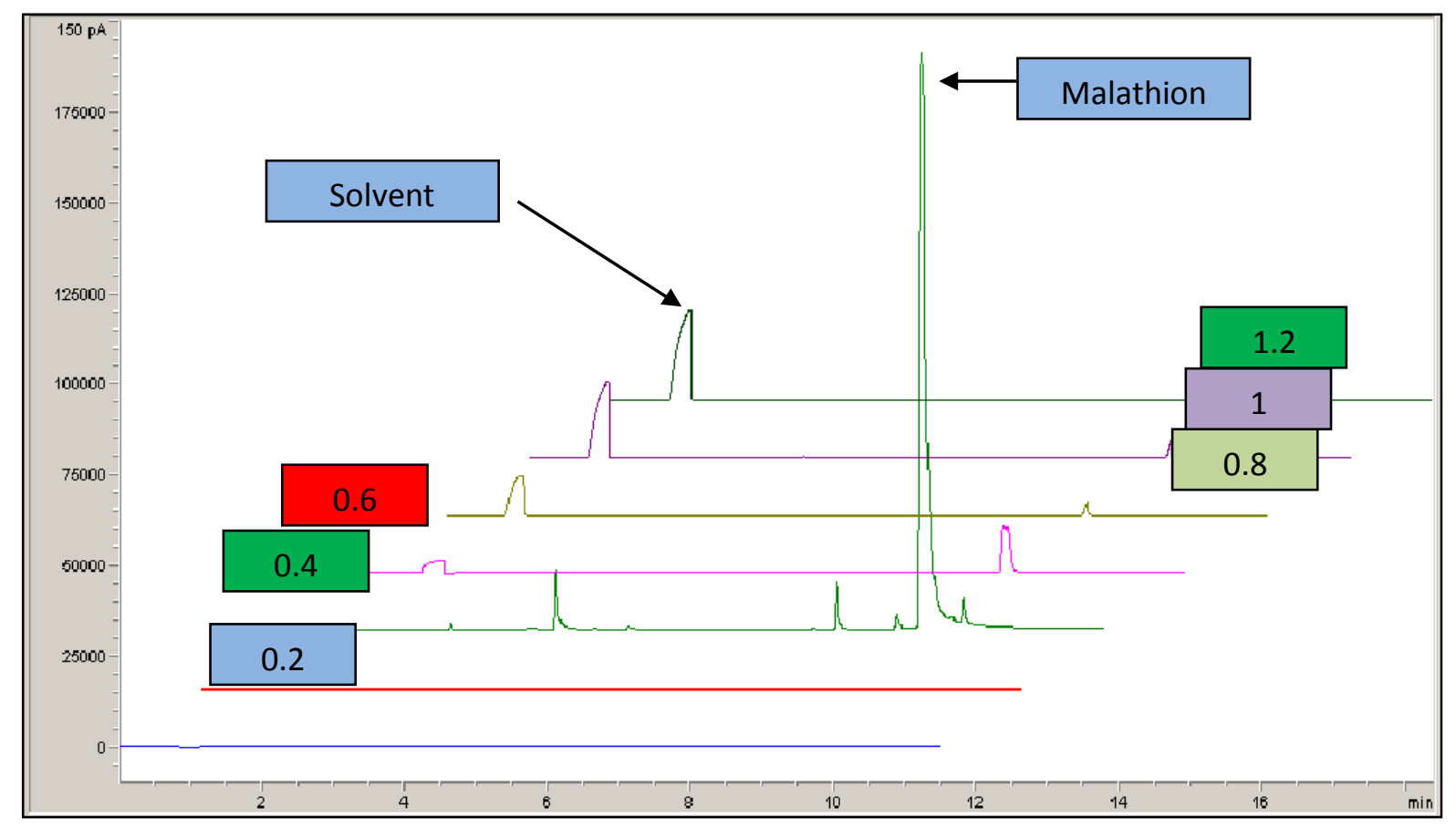

Fig. 5. 3D chromatograms of different air to $\mathrm{H}_{2}$ ratio GC-FPD-P mode for Malathion, measured at GC operating condition.

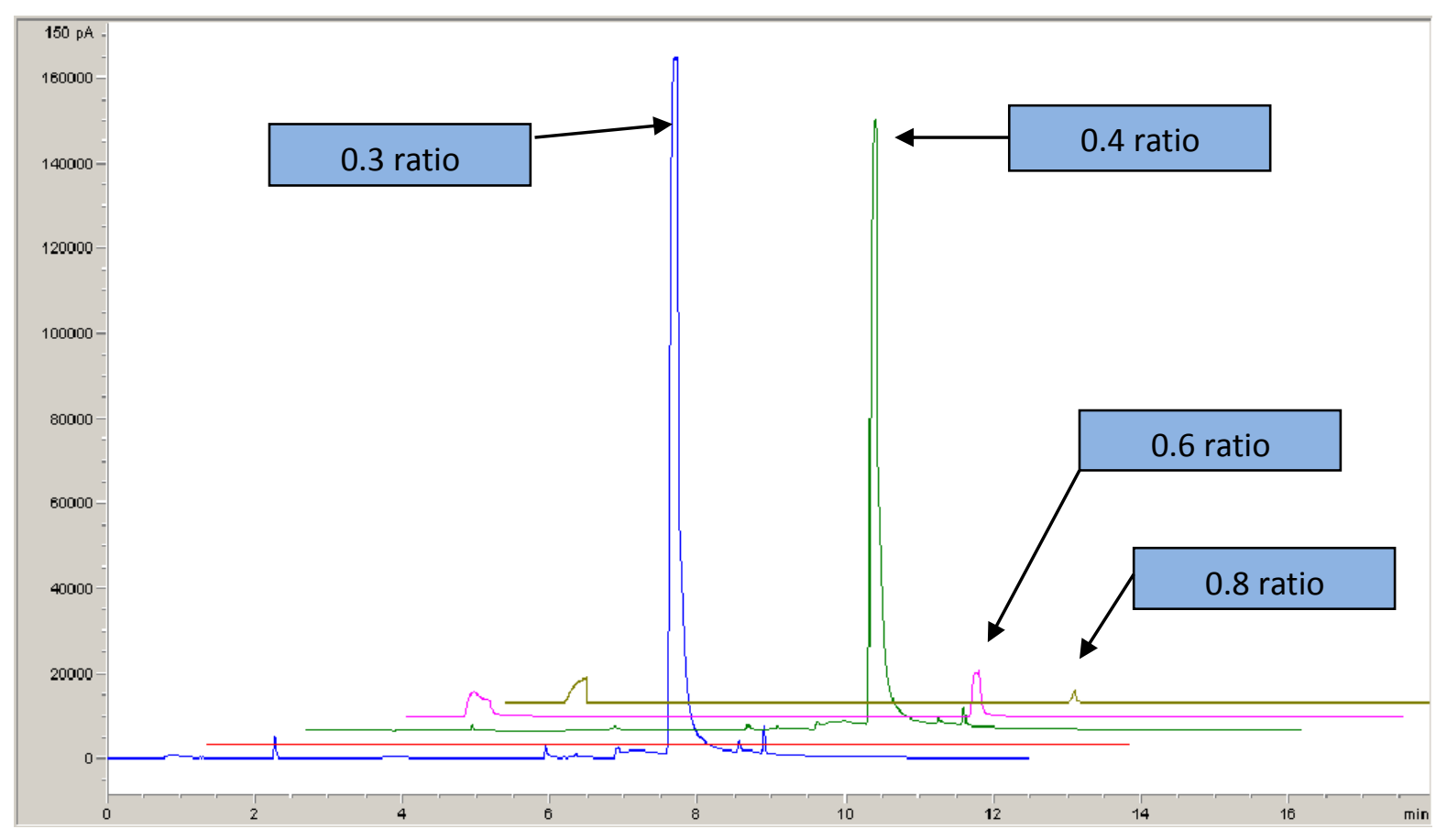

Fig. 6. 3D chromatograms of different air to $\mathrm{H}_{2}$ ratio GC-FPD-P mode for Dimethoate, measured at GC operating condition. 


\section{2. Effect of total gases flow rates in the performance of FPD-P mode}

In this type of measurements, the total gas flow rate was varied from 105 to $210 \mathrm{ml} / \mathrm{min}$ at air to hydrogen ratio 0.4 for Malathion and from 65 to $195 \mathrm{ml} / \mathrm{min}$ at air to hydrogen ratio 0.3 for Dimethoate. Detector responses were determined at different total gas flow rate and the results are shown in Figures 7 and 8 for both Malathion and Dimethoate, respectively. Figures 9 and 10 shows the 3D chromatograms of detector responses verses total gas flow rates for Malathion and Dimethoate, respectively. As indicated from the figures, the performance increases with increasing total flow rates.

This can be attributed to Hydrogen - Air or Hydrogen - Oxygen diffusion flames are normally employed for the FPD. In such diffusion flames, the hydrogen and oxygen do not mix instantaneously, so that these flames are characterized by significant spatial variations in both temperature and chemical species. The important chemical species in hydrogen - air flame are the $\mathrm{H}, \mathrm{O}$, and $\mathrm{OH}$ flame radicals.

These highly reactive species play a major role in decomposing incoming samples and in the subsequent production of the desired optical emissions. Optical emissions from the HPO molecular systems are highly favored in those regions of an FPD flame which are locally rich in $\mathrm{H}$-atoms, while $\mathrm{CH}$ and $\mathrm{C}_{2}$ light emissions from hydrocarbons originate mainly from those flame regions which are locally rich in O-atoms.

The highest sensitivity and specificity for phosphorus detection are achieved only when the FPD flame is operated with hydrogen in excess of that stoichiometric amount required for complete combustion of the oxygen supplied to the flame. This assures a large flame volume that is locally abundant in H-atoms, and a minimal flame volume that is locally abundant in O-atoms, [11].

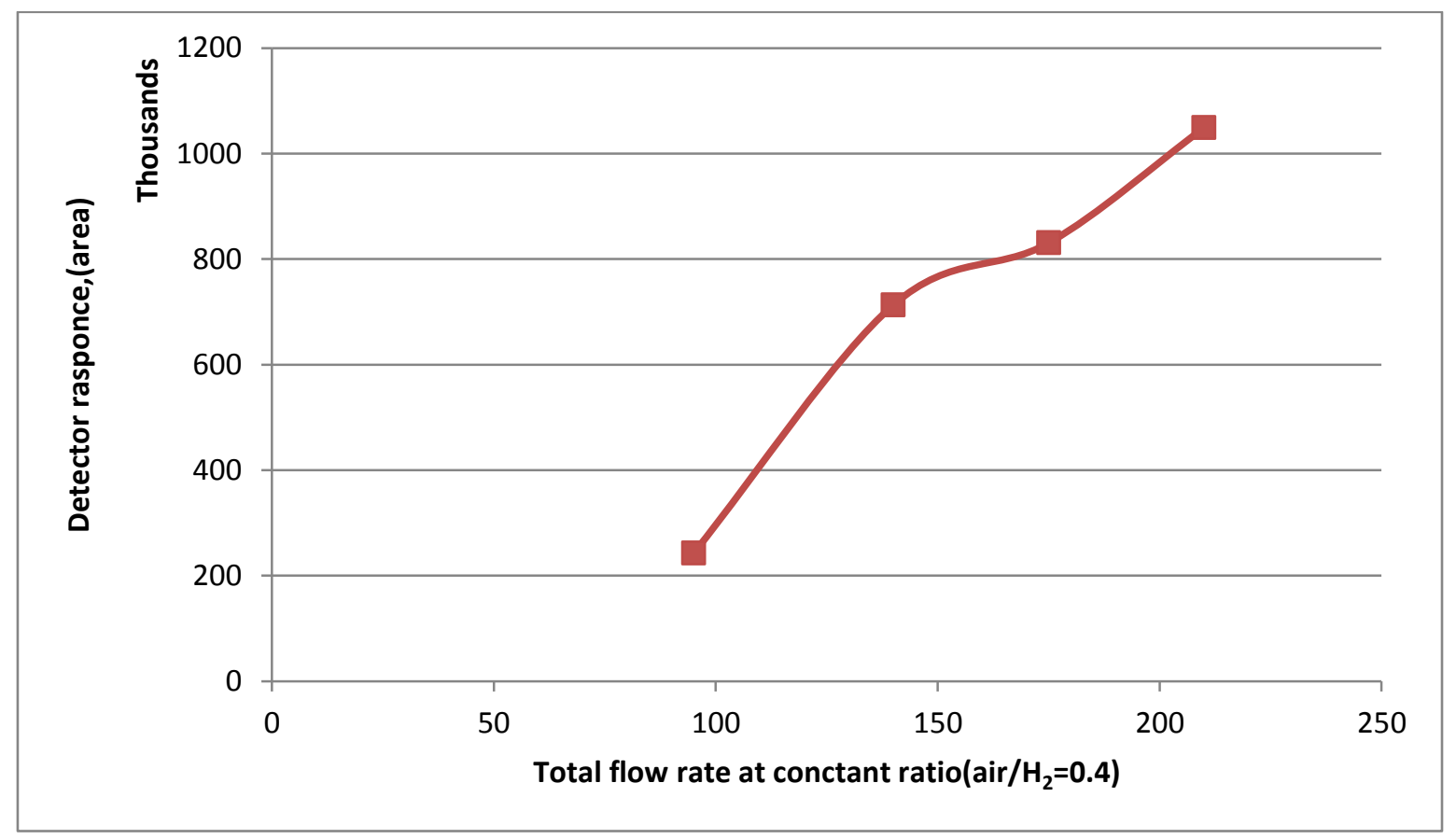

Fig. 7. Detector responses versus total gases flow rate for Malathion GC-FPD-P-mode, at the same operating condition. 


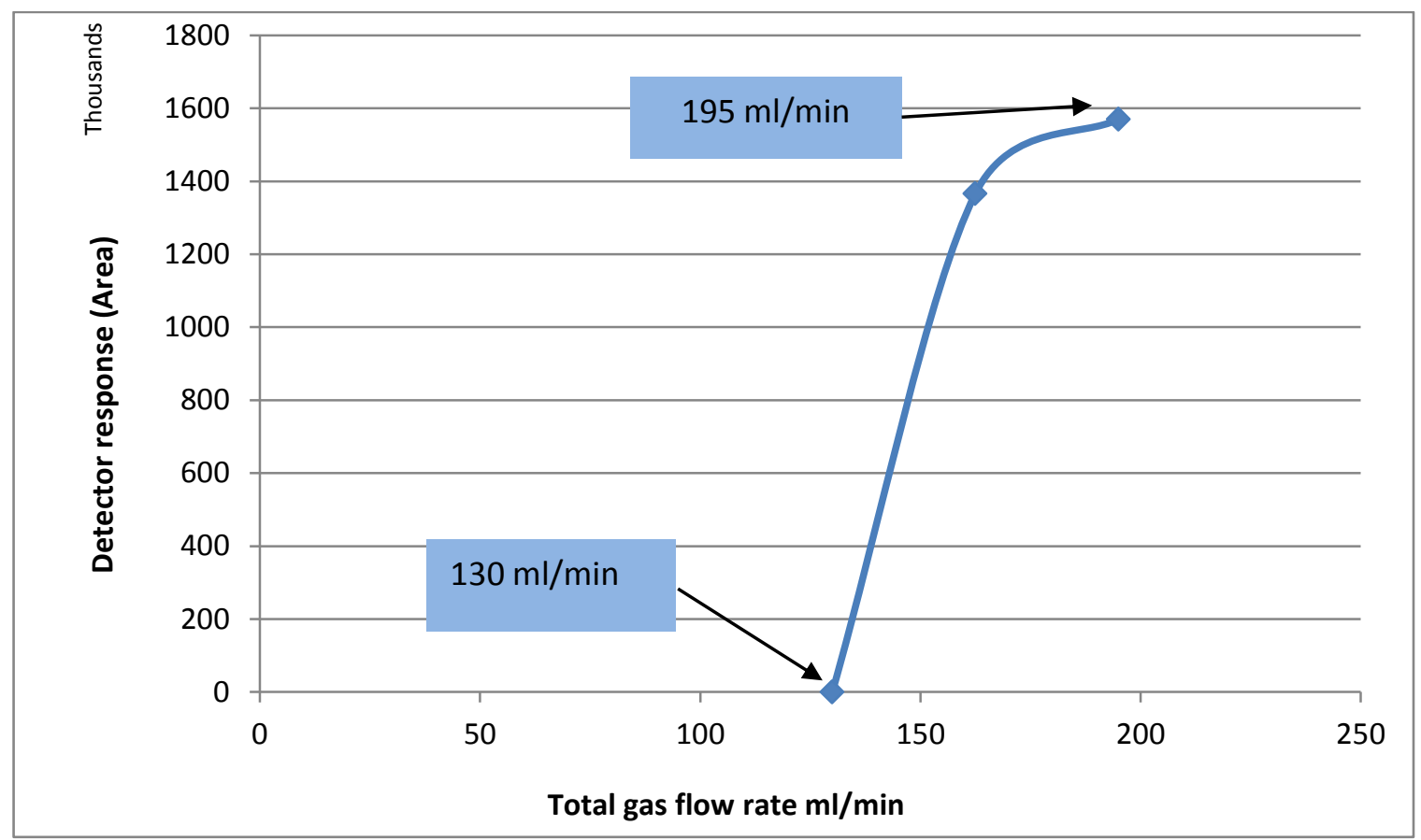

Fig. 8. Detector responses versus total gases flow rate for Dimethoate GC-PFD-P mode, at the same operating condition.

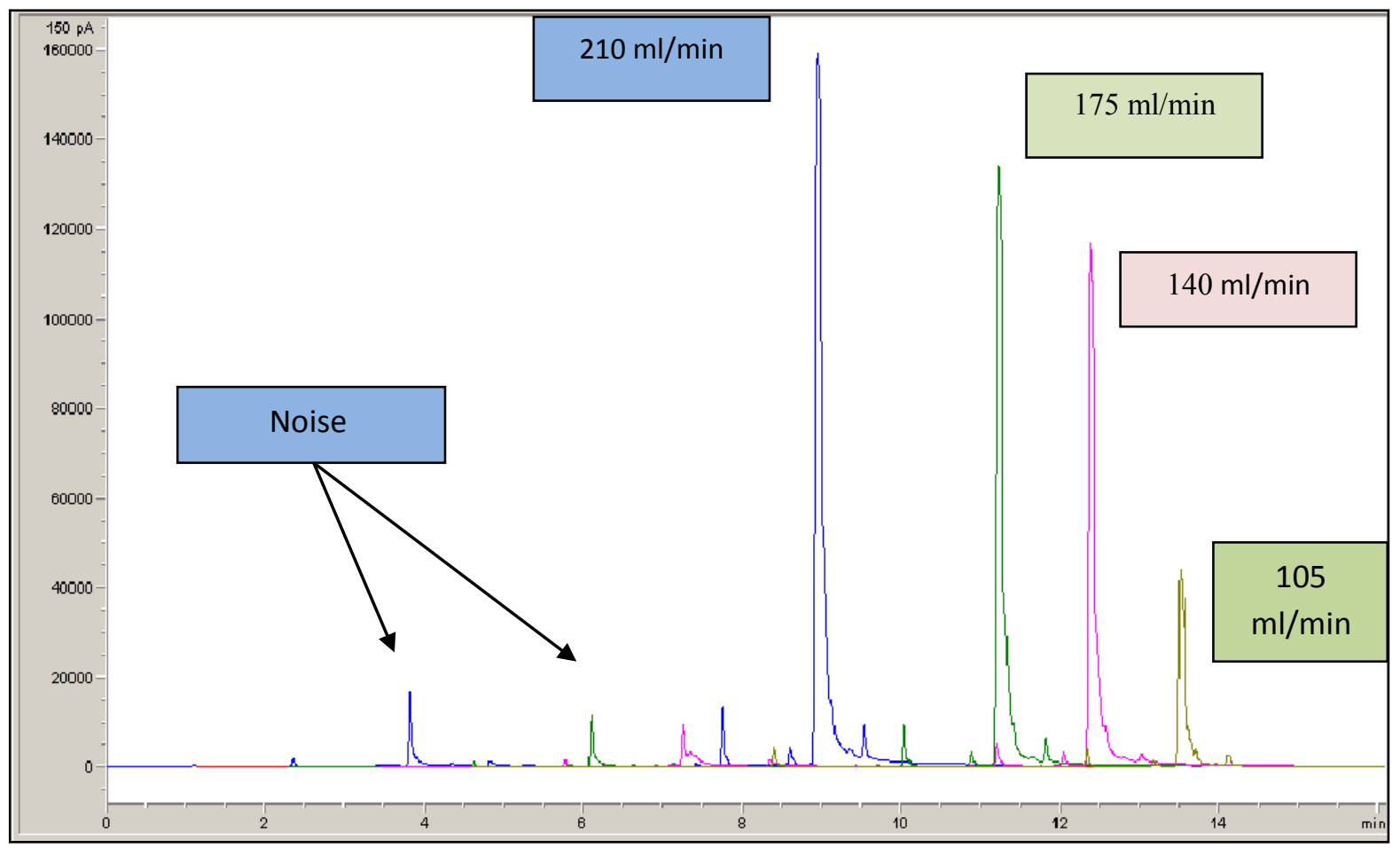

Fig. 9. (3D) Chromatograms of detector responses versus total gases flow rates for Malathion, at the same operating condition. 


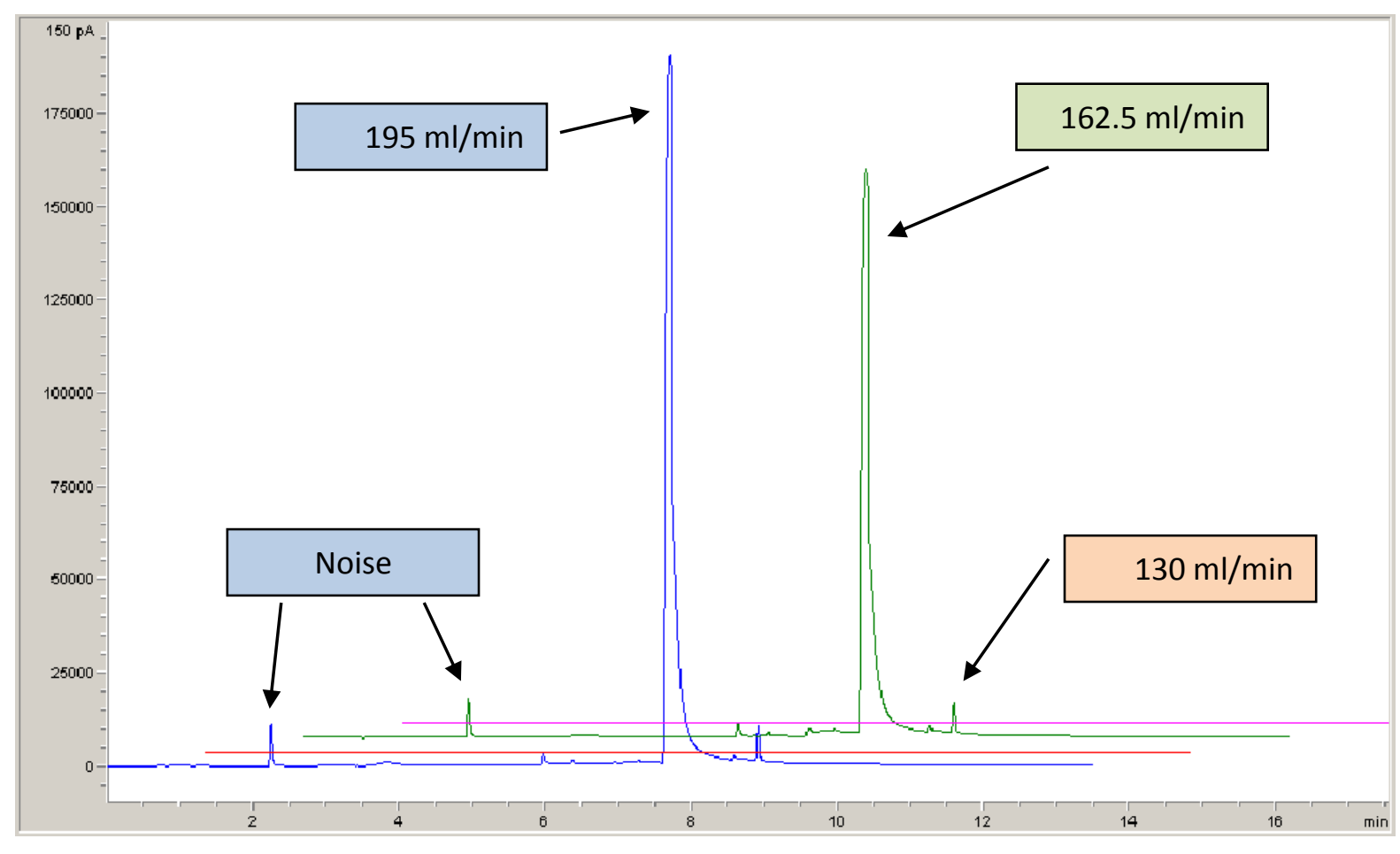

Fig. 10. (3D) Chromatograms of different total gases flow rate for Dimethoate, at the same operating condition.

\section{3. Linearity of FPD-P mode with Malathion and Dimethoate}

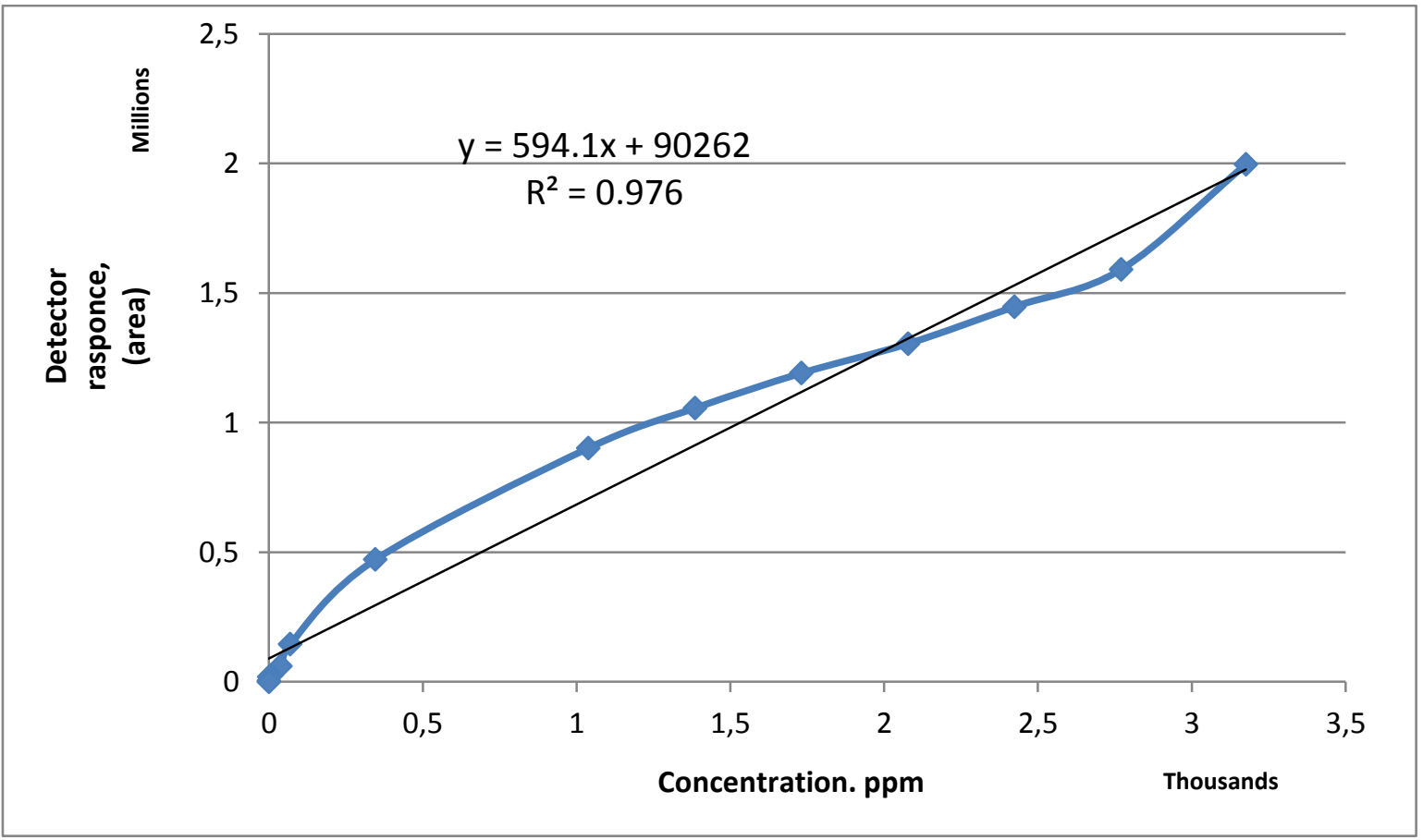

Fig. 11. Linear range of the detector (FPD-P-mode) for Malathion measured at optimum operating conditions. 
The linearity of the detector FPD-P mode was determined by the injection of $1 \mu \mathrm{L}$ standard solution at different concentrations. Calibration curves plotted from different concentrations were obtained for analytes by regression analysis of peak area versus injection concentration.

Figures 11 and 12 shows the relation between detector response (area under the curve) and concentrations of pesticide. The plot shows the linearity of the detector with Malathion and Dimethoate with $R^{2}$ value higher than 0.997 over the studied concentrations range, which indicate good linearity. Similar results for $R^{2}(0.999-0.9823)$ were obtained in a study using GC/PFPD and GC/MS for the determination of trace OPPs, [12-14].

Figures 11 and 12 presented the determined linearity of the detector with Malathion and Dimethoate, respectively. The linear range of the detector for Malathion covers the concentration range from $40.2 \times 10^{-3}$ to $3175.819 \mathrm{mg} / 1$ with correlation coefficient value $\left(\mathrm{R}^{2}\right)$ $=97.6 \%$ while it was from 3.8 to $638.005 \mathrm{mg} / 1$ with correlation coefficient value $\left(\mathrm{R}^{2}\right)=$ 97.2\% for Dimethoate



Fig. 12. Linear range of the detector (FPD-P-mode) for Dimethoate measured at optimum operating conditions.

\section{4. Accuracy and precision measurement of the method for Malathion and Dimethoate}

The precision and accuracy of the methods were evaluated using relative standard deviation for replicates $(n=5)$. Standard concentrations of 1385.273 and $315.439 \mathrm{mg} / \mathrm{l}$ for Malathion and Dimethoate, respectively were injected 5 times, the results are shown in Table 1 and 2. The relative standard deviation of the peak areas of the pesticides were (RSD) $=$ $1.979 \%$ and $0.363 \%$ for Malathion and Dimethoate, respectively. The result revealed that, the deviation was not exceeding $20 \%$, in accordance with the methods of validation of pesticide residues reported in other research [15]. In addition to, the deviation was in a range similar or better than those obtained in other studies using GC-FPD-P mode (1.7, 1.2, and 5.6 $\%)$ [16]. 
Table 1. Five injection of Malathion in GC-FPD-P-mode.

\begin{tabular}{|c|c|c|}
\hline Sample no. & Area (150pA/s) & $\begin{array}{c}\text { Conc. according to } \\
\text { calibration curve } \\
(\mathrm{mg} / \mathrm{l})\end{array}$ \\
\hline 1 & 1056032.4 & 1625.602 \\
\hline 2 & 1051107.6 & 1617.313 \\
\hline 3 & 1060550.9 & 1633.208 \\
\hline 4 & 1062909.7 & 1637.178 \\
\hline 5 & 966233.9 & 1474.452 \\
\hline Mean & 1039366.9 & 1597.551 \\
\hline Accuracy & \multicolumn{2}{|c|}{$15.3 \%$} \\
\hline Precision & \multicolumn{2}{|c|}{$1.979 \%$} \\
\hline
\end{tabular}

Table 2. Five injection of Dimethoate in GC-FPD-P-mode.

\begin{tabular}{ccc}
\hline Sample no. & Area (150pA/s) & $\begin{array}{c}\text { Conc. according to } \\
\text { calibration curve }\end{array}$ \\
\hline 1 & 823896.1 & 341.6105 \\
2 & 825796.1 & 342.2671 \\
3 & 830050.6 & 344.3853 \\
4 & 825316.7 & 342.2509 \\
5 & 827216.7 & 343.1076 \\
Mean & & 342.7243 \\
Accuracy & & $8.6499 \%$ \\
Precision & & $0.363 \%$ \\
\hline
\end{tabular}

\section{5. Determination of Limit Of Detection (LOD) and Limit Of Quantification (LOQ) for the detector GC-FPD-P mode for Malathion and Dimethoate}

LOD and LOQ are measured for the methods by the analysis of ultra-pure samples (Malathion and Dimethoate) with decreasing concentrations until reach the lowest detectable level of the organophosphorus pesticides that could be detected by the GC-FPD-P mode.

The values of LOD were found to be $1.88 \times 10^{-3} \mathrm{mg} / 1$ and $1.52 \mathrm{mg} / 1$ for Malathion and Dimethoate, respectively these concentrations equal to three times the signal/noise ratio. On the other hand, the lowest determinable levelS (LOQ) were $0.017 \mathrm{mg} / \mathrm{l}$ and $5.7 \mathrm{mg} / \mathrm{l}$ for Malathion and Dimethoate respectively, these concentrations equal to ten times the signal/ noise ratio. Figure 13 and 14 shows LOD and LOQ for Malathion. The method show good sensitivity for the detection of Malathion compared to Dimethoate, this may be due to the differences in the chemical and physical properties of both compounds and their interaction with the stationary phase of the column. 


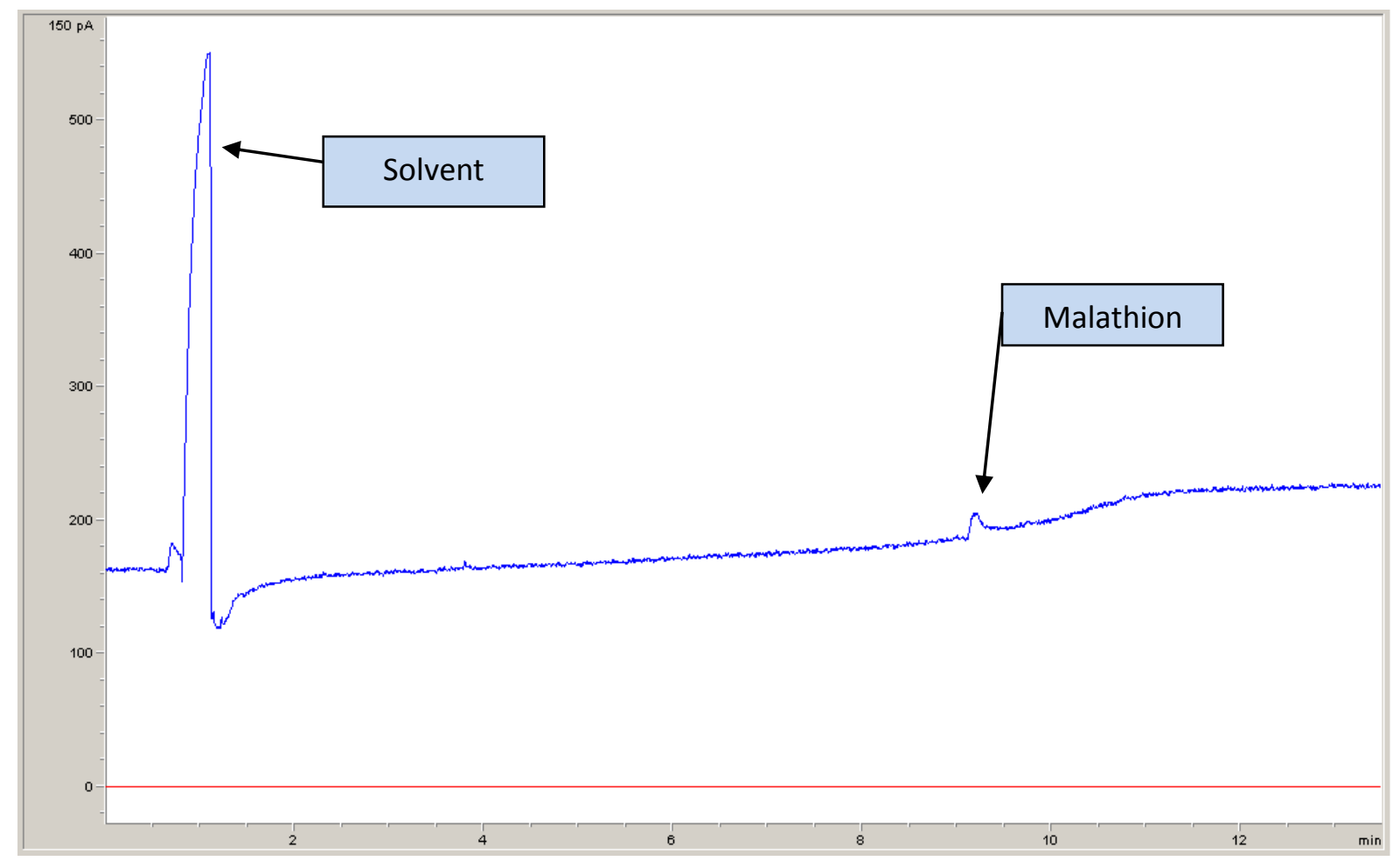

Fig. 13. Chromatogram of $1.88 \times 10^{-3} \mathrm{mg} / 1$ Malathion (LOD), at optimized conditions.



Fig. 14. Chromatogram of $0.017 \mathrm{mg} / 1$ Malathion (LOQ), at optimized condition 
The samples components are usually held on the stationary phase so strongly that they are eluted very slowly or even not at all [6]. In this method, the used column is HP-5 with non polar stationary phase which is favor the non polar compounds such as Malathion, while it is not favor the compounds have polarity such as Dimethoate.

\section{CONCLUSIONS}

In conclusion, the study of air-to-fuel ratio with flame photometric detector for the analysis of Malathion and Dimethoate samples showed the validity of Gas ChromatographyFlame Photometric Detector (P and S -modes) providing excellent separation and peak shape. From the results carried out for the analysis in this study, it was revealed that, the method applied using GC-FPD, the optimum air to hydrogen ratio is at regions of the FPD flame which are locally rich in $\mathrm{H}$-atoms such as 0.4 for the analysis of Malathion and 0.3 for the analysis of Dimethoate with P mode and 0.6 with S-mode. The highest sensitivity and specificity for phosphorus detection are achieved only when the FPD flame is operated with hydrogen in excess of that stoichiometric amount required for complete combustion of the oxygen supplied to the flame. The method is precise, reproducible, simple, rapid, has a wide range of linearity and dynamic range over a wide range of analyte concentrations with correlation coefficient $\left(\mathrm{R}^{2}=0.968\right)$ and $(\mathrm{RSD})<4.5 \%$, and uses small volumes of solvents and samples, which reducing the risk for human health and the environment. Besides, the results of this study show that the proposed method could be further applicable to determine the residues of different pesticides in environment.

\section{References}

[1] Bowman M., M. Beroza, Journal of Chromatographic Science 7(8) (1969) 484-492.

[2] Crider W. L., Analytical Chemistry 41(3) (1969) 534-537.

[3] Bennett G. F., Introduction to Environmental Analysis: Roger Reeve, John Wiley \& Sons, Inc., New York, MY, 2002, Elsevier.

[4] Brody S. S., J. E. Chaney, Journal of Chromatographic Science 1966. 4(2) (1966) 42-46.

[5] Versino B., G. Rossi, Chromatographia 4(8) (1971) 331-336.

[6] Dagnall R., K. Thompson, T. West, Analyst 93(1103) (1968) 72-78.

[7] Marek B., Todd S., Aamand S., Journal of Enzyme Inhibition and Medicinal Chemistry 18(6) (2003) 551-555.

[8] WHO, "Malathion in Drinking water", 2004, WHO/SDE/WSH/103.

[9] A. Ayman, Gouda Alaa S., Amin Ragaa El Sheikh, Chemical Industry \& Chemical Engineering Quarterly 16(1) (2010) 11-18.

[10] WHO. Environmental Health Criteria monograph "Dimethoate", WHO, Geneva, 1989.

[11] Dömötörová M., E. Matisová, Journal of Chromatography A 1207(1) (2008) 1-16.

[12] Wang D., D. P. Weston, M. J. Lydy, Talanta 78(4) (2009) 1345-1351.

[13] Saeed T., et al., Food Control 12(2) (2001) 91-98. 
[14] Frishman G., A. Amirav, Field Analytical Chemistry \& Technology 4(4) (2000) 170-194.

[15] Bidari A., et al., Food Chemistry 126(4) (2011) 1840-1844.

[16] Samadi, S., H. Sereshti, and Y. Assadi, Journal of Chromatography A 1219 (2012) 61-65. 